Estudios Románicos, Volumen 28, 2019, pp. 389-402

ISSN: 0210-491

eISSN: 1989-614X

DOI: https://doi.org/10.6018/ER/360591

\title{
EL ROSA PEDAGOGICO ITALIANO EN LA PRIMERA MITAD DEL SIGLO XX: LAS SEÑORITAS DE WANDA BONTÀ
}

(The pedagogical purpose in romance novels during the first half of the 20th Century: Wanda Bontà's "Signorinette")

\author{
Anna Suadoni* \\ Universidad de Granada
}

\begin{abstract}
From the twenties the romance fiction acquires the proportions of a publishing phenomenon, written, administered and read by women. In Italy many romantic plots become carriers of an educational message that aims to instruct the readers to become perfect mothers and wives, fitting in the place that corresponds to them in society. However, in many of these works, stories and female figures often reveal the conflict between traditional models and wishes of emancipation.
\end{abstract}

Keywords: Popular literature; romance novels; women's writing; fascist society; female stereotypes; female emancipation.

Resumen: A partir de los años veinte del siglo pasado, el sector del género rosa adquiere las proporciones del fenómeno editorial, escrito, administrado y leído por mujeres. En Italia muchas tramas románticas se hacen portadoras de un mensaje educativo que pretende instruir a las lectoras para llegar a ser madres y esposas perfectas y encontrar así el sitio que les corresponde en la sociedad. Sin embargo, en muchas de estas obras, las historias y las figuras femeninas revelan a menudo el conflicto entre modelos tradicionales y deseos de emancipación.

Palabras clave: Literatura popular; literatura rosa; literatura femenina; sociedad fascista; estereotipos femeninos; emancipación femenina.

*Dirección para correspondencia: Anna Suadoni. Departamento de Filologías Románica, Italiana, Gallegoportuguesa y Catalana. Facultad de Filosofía y Letras. Campus Cartuja.18071 Granada (asuadoni@ugr.es). 


\section{La novela rosa}

Es complicado identificar características formales y estructuras narrativas típicas del género rosa, que, como en el caso de la literatura infantil, selecciona su público "non attraverso una vocazione di lettura (magari caratterizzata da precisi connotati sociologici), ma attraverso dati anagrafici come l'età o il sesso" Rocella (1998: 4). De hecho la literatura rosa parece definirse exclusivamente por el tipo de argumento que incluye una historia romántica.

En la primera mitad del siglo xx la novela rosa y, más en general, la literatura destinada a un público exclusivamente femenino es un fenómeno editorial muy extendido que se desarrolla entre publicaciones por entregas y novelas en edición económica realizadas por un ejército de editores y escritoras, "tanto che una ricognizione fra le firme del rosa italiano, soprattutto intorno agli anni Cinquanta, che avesse pretese di fornire un catalogo esaustivo, diventerebbe una ricerca labirintica e infinita" (Rocella 1998: 75).

De hecho, ni siquiera la definición del género puede prescindir de su relación con la industria editorial. Anna Banti, que en 1953 publica en Paragone ${ }^{1}$ un breve y muy citado ensayo sobre el género sentimental, afirma a propósito de las escritoras rosa:

Giova a questo punto considerare le persone delle scrittrici. Conscie, la più parte, della barriera che le divide dalla letteratura propriamente detta, son le prime a trascurare la loro notorietà, e a far capire, in ogni occasione, di considerarsi impresarie di un'industria redditizia e di grande smercio (Banti 1953: 30).

Pozzato, en relación a la cita de Banti, escribe que: "Il genere rosa si definisce dunque un genere commerciale, d'evasione, non letterario o, come dicono appunto alcuni, paraletterario" (Pozzato 1982: 12).

Al principio del siglo xx, la novela rosa no había conseguido el mismo éxito que otros géneros en las publicaciones por entregas, las cuales habían logrado alcanzar las clases sociales más bajas gracias a una prosa adecuada a su nivel cultural y a un precio accesible que permitía repartir en plazos el coste demasiado alto de un libro entero ${ }^{2}$. Dadas las dimensiones del mercado, las editoriales de publicaciones por entregas se habían organizado a nivel internacional y en muchos casos las mismas historias eran traducidas y distribuidas en distintos países. Para satisfacer el gusto de un público tan amplio, empiezan a aparecer en este periodo los distintos géneros paraliterarios (Detti 1989: 45): western, policíaco, aventura, etc.

Sin embargo las historias con temática sentimental no conseguían apasionar a los lectores de las entregas. Ello es debido a que a partir del siglo XVIII el final feliz,

$1 \quad$ Paragone es una revista bimensual de arte y literatura fundada por Anna Banti y Roberto Longhi en 1950. Está organizada en dos series dedicadas al arte y a la literatura. Longhi dirigirá la serie de arte hasta su muerte, en 1970. Banti se ocupará de la serie literaria, junto a un comité formado por un grupo de intelectuales del tiempo (Attilio Bertolucci, Piero Bigongiari, Carlo Emilio Gadda, Adelia Noferi, Giorgio Bassani y Giorgio Zampa) cuya presencia demuestra el compromiso de la revista con las nuevas tendencias literarias (Mirabile 2005).

2 De hecho, en Estados Unidos, las publicaciones periódicas se llamaban dime novels (novelas de diez centavos), haciendo referencia tanto a la calidad literaria, cuanto, literalmente, al precio. 
con la unión de los protagonistas, se vuelve un elemento imprescindible de este tipo de narración. Este está vinculado esencialmente con el comportamiento de la mujer: solo si esta sabe conservar su propia virtud y defenderse de los halagos del hombre, podrá aspirar al matrimonio. De hecho, el origen de la novela rosa se remite al 1739, con Pamela o la virtud recompensada, de Samuel Richardson, historia de una joven criada que, resistiendo a las atenciones del padrón, consigue finalmente casarse con él. Si en aquella época la literatura popular era ya muy difusa, Pamela es probablemente el primer intento de dirigirse exclusivamente a un público femenino (Detti 1989: 40).

Así que las historias de amor tenían que acabar con un matrimonio y esto impedía proponer dos veces las aventuras de los mismos personajes, cuya rutina matrimonial no habría despertado ningún interés. Cada vez que una historia de amor terminaba, había que empezar una nueva con otros personajes negando a las lectoras la posibilidad de encariñarse con ellos.

Por tanto empiezan a circular, en Italia como en otros países, fascículos independientes de pocas páginas, relatos de amor en edición muy económica pero con un diseño cautivador. Las historias podían estar traducidas de otros idiomas o, más a menudo, escritas por autores italianos desconocidos, normalmente bajo un seudónimo extranjero. En 1926, la editorial Picco de Turín publica semanalmente pequeños libros de cuarenta páginas que siguen un esquema editorial fijo: misma extensión, misma estructura narrativa y títulos que aluden explícitamente a la trama romántica.

En los mismos años, de hecho, el género rosa empieza a perfilarse como un producto editorial moderno. Algunas editoriales, como Salani o Sonzogno, se especializan en literatura de consumo y diversifican su oferta creando colecciones. La edición llega a ser una verdadera industria y se buscan productos de gran difusión. Muchas revistas destinadas a mujeres (Anabella, Rakam, Gioia, Grazia ${ }^{3}$, entre otras) nacen en este periodo. Dentro de esta especialización de la producción editorial se forma un sector rosa, dirigido, escrito y leído por mujeres. Se perfila la figura de la escritora/periodista y en algunos casos, Wanda Bontà por ejemplo, también directora de revistas. Las escritoras más conocidas (Wanda Bontà, Liala, Mura, Luciana Peverelli, Giana Anguissola ${ }^{4}$ ) son, por lo general, muy prolíficas y comprometidas en varias labores editoriales (novelas, relatos en revistas, columnas de consejos a las lectoras)

3 Annabella, fundada en 1933, era una revista semanal publicada por la editorial Rizzoli que tuvo entre sus colaboradores a Giorgio Scerbanenco (La posta di Adrian) y Brunella Gasperini (Ditelo a Brunella). En 1938 Mondadori empieza la publicación de Grazia, revista de moda que contará con las colaboraciones de Irene Brin y Colette Rosselli, entre otras. Rakam es una revista mensual de "labores femeninas" fundada en 1930 y publicada inicialmente por L'iniziale (Milán) y luego por Rusconi. El mismo recorrido editorial tuvo la revista semanal femenina Gioia, fundada en 1937 (De Feo 2012).

4 Mondadori creó una revista semanal para Liala: Le confidenze di Liala, luego llamada Confidenze; Mura empezó su carrera como periodista, sobre todo para L'Illustrazione Italiana, y trabajó mucho escribiendo crónicas de sus viajes alrededor del mundo, además de ser traductora de francés y de publicar libros infantiles; Luciana Peverelli, como Wanda Bontà, fue una asidua colaboradora de Cino Del Duca dirigiendo varias revistas y colaborando con otras (Il Monello, Stelle, Grand hotel, Stop, entre otras); fue también traductora de inglés. Giana Anguisola trabajó como periodista, siendo incluso enviada especial para la Gazzetta Lombarda (Pozzato 1979; Antonutti 2015; Åkerström 2017; Wintersgill 2004). 
Las proporciones del mercado editorial y su estatus de literatura de consumo no favorecen la relación con la crítica que ha sido desde el principio muy conflictiva. Benedetto Croce (1940: 185) habla de "un infinito pulviscolo di romanzatrici, le instancabili romanzatrici" por un lado refiriéndose a datos objetivos como la gran dimensión de la producción literaria, o el hecho de que esté en manos exclusivamente de escritoras, y por otro insinuando un juicio negativo sobre la falta de calidad literaria que implica satisfacer un mercado tan amplio (Rocella 1998: 20). Escritoras como Carolina Invernizio, considerada pionera del género, o Liala, probablemente la más famosa de las autoras de rosa, comparten un enorme éxito de público y el desdén de la crítica literaria: Gramsci llama a Invernizio "onesta gallina” (Gramsci 1975: 344) y el Gruppo 635 definirá "Liale" con irónico desprecio a autores como Bassani, Pratolini e Cassola.

La crítica de Anna Banti a propósito del género sentimental también es durísima: "[...] noi ci eravamo proposti di esaminare quella del romanzo di gran consumazione e che non sarebbe più il caso di chiamare romanzo rosa, ma forse grigio: il colore della velocità, della folla e anche della disattenzione [...]" (Banti 1953: 28). Y más adelante:

Il romanzo, gloria del secolo decimonono, minacciava le basi della società borghese. Padri benpesanti e direttori spirituali non riparavano a condannare, a proibire, e fu con vero sollievo che si videro comparire certi libretti dalla copertina rosa o celeste, abbondantemente ornati di fregi e di incisioni. Erano sciocchi, erano, magari, sgrammaticati, ma ci si poteva fidare: leggessero, le dame e damigelle, se proprio non potevano farne a meno (Banti 1953: 29).

\section{El rosa trasgressivo y el rosa pedagogico}

El canon narrativo del género rosa se va perfilando poco a poco. En la gran caldera de las publicaciones sentimentales de principios de los años veinte, muy desiguales por calidad literaria y estilo, se definen dos "corrientes": el rosa trasgressivo y el rosa pedagogico (Rocella 1998: 37). Liala (seudónimo, elegido por D'Annunzio, de Amalia Liana Negretti Odescalchi) y sobre todo Mura (Maria Volpi Nannipieri) son las principales representantes del primero (Åkerström 2017). En las obras de estas autoras junto a la función evasiva, implícita en el tipo de producto editorial, se puede encontrar cierta sensualidad, aunque muy pudorosa. Liala y Mura se hacen portavoces del deseo femenino y si bien evitan argumentos tabúes como libertinaje, homosexualidad y prostitución (Ricci 2017: 90), ofrecen a las lectoras historias y sobre todo modalidades textuales (como las descripciones físicas de los héroes masculinos que persisten en detalles bastantes íntimos) que evocan claramente la atracción erótica (Rocella 1998 y Ricci 2017).

La audacia absolutamente novedosa de las historias de Liala tiene un enorme éxito de público y convierte a esta escritora en la firma por excelencia del rosa italiano. No obstante, a pesar de que Liala sea su mayor exponente, el rosa italiano se decanta fundamentalmente por una función de tipo pedagógico:

$5 \quad$ El Gruppo 63 fue un movimiento literario de neovanguardia; se constituye en Palermo en octubre de 1963 y opone a la "ultima fiammata del neorrealismo" una nueva literatura que, a través de un experimentalismo lingüístico extremo, sepa reflejar la nueva realidad social del boom económico (Balestrini; Giuliani; Barilli; Guglielmi 2013). 
La tendenza realista è connaturata al rosa italiano, con l'eccezione di Liala. Pur avendo canonizzato il genere, Liala in un certo senso non è riuscita a fare scuola, proprio per l'invincibile vocazione al realismo e alla pedagogia presente in tante scrittrici rosa (Rocella 1998: 82).

También Faeti (1980) identifica con la vocación pedagógica la característica principal del rosa italiano: "Il rosa infatti colora, con coerente uniformità di toni pedagogici, prodotti che risultano assai diversi per ampiezza, dignità letteraria e caratteristiche del medium usato per raggiungere i fruitori" (Faeti 1980: 135).

En las obras de escritoras como Wanda Bontà, Giana Anguissola o Luciana Peverelli destaca la aspiración edificante y moralista (Sani 2014), a veces claramente alineada con el régimen fascista. Su objetivo fundamental, como en el caso de casi todos los productos editoriales para las mujeres, además de entretener, es educar. Y si las revistas ofrecen consejos para formar a buenas amas de casa y madres de familia, en las historias de amor el mensaje es muy claro: portarse como es debido y dar muestras de sentido común premia a la protagonista con un buen matrimonio. El amor romántico vivido como un sueño, presente en las historias de Liala, deja el paso al realismo: el matrimonio es necesario para que una mujer encuentre su espacio en la sociedad y pueda alcanzar la seguridad económica.

En realidad, el público femenino busca y encuentra siempre en la novela rosa modelos de comportamiento que pueden ser convencionales, como en el rosa pedagogico, o anticonformistas, como los ofrecidos por las obras más transgresivas:

Il romanzo rosa è sempre, in un modo o nell'altro, un romanzo di formazione al femminile per giovani donne piene di incertezze, di dubbi a cui nessuno risponde, oppresse da modelli ancora assai rigidi ai quali cominciano a contrapporsi, però, contraddittori segnali che vengono dall'esterno. (Rocella 1998: 37).

Esta función formativa que asumen los productos editoriales dedicados a las mujeres se hace clara en las columnas de consejos en las revistas femeninas, en muchos casos gestionadas por escritoras de rosa.

La literatura rosa y sus autoras llegan a ser un punto de referencia para la formación de las lectoras y el rosa pedagogico, a pesar de su misión educativa y los intentos de moderar los aspectos transgresivos de las relaciones sentimentales, se hace también portavoz del conflicto entre el deseo de modernización y emancipación de la mujer y su papel tradicional en la sociedad.

\section{Wanda Bontà}

Representante destacada del rosa pedagogico, Wanda Bontà (Milán 1902-1986) empieza a escribir cuando las publicaciones románticas han encontrado finalmente un espacio en el mercado editorial (Morreale 2011; Bravo 2011; Sani 2014). 
Existe poquísima bibliografía sobre su obra y muy pocas noticias sobre su biografía. Estas últimas perfilan un recorrido formativo y profesional muy típico en este periodo para una joven mujer que tuviera la posibilidad de estudiar: aprende estenografía, obtiene el título para enseñar en la escuela primaria y trabaja como monitora en las escuelas de verano. Es decir, una formación como secretaria o maestra. Paralelamente, se dedica a la escritura. Ella misma, en la introducción a la edición de 1969 de Signorinette, nos cuenta que escribía por las noches, para evadirse de la soledad y de las normas rígidas impuestas por las residencias en las que le tocaba vivir por ser huérfana (Bontà 1969). En 1928 publica su primera novela, parcialmente autobiográfica, La fatica di vivere, que obtiene cierto éxito de crítica y de público y que le permite dedicarse exclusivamente a la escritura. A partir de este momento, colabora con varias revistas femeninas escribiendo relatos, novelas por entregas y llevando columnas de consejos.

Gracias a su conocimiento del mercado editorial femenino, en 1937 es llamada a dirigir L'intrepido, revista de cómic publicada por la editorial Universo. Empieza así la colaboración con los editores Del Duca ${ }^{6}$ que sigue después de la segunda guerra mundial con el trabajo parados revistas semanales de gran difusión, Intimità e Grand Hotel, donde se ocupa de una columna de consejos muy conocida, Filo d'oro.

En 1938, un editor le encarga un libro per giovanette. Según lo que nos cuenta la misma escritora (Bontà 1991:8), la petición llega en octubre y el plazo de entrega es diciembre, para la campaña comercial de Navidad. Así nace Signorinette, que en poco tiempo alcanza las trece ediciones y que inspirará la homónima película de Luigi Zampa en 1942. El mismo año, en plena guerra mundial, Bontà publica Signorinette nella vita en el que las protagonistas de Signorinette, terminados sus estudios, empiezan a enfrentarse con las dificultades, profesionales y sentimentales, de la vida adulta.

Entre 1940 y 1943 aparece como columna semanal en la revista Grazia (Mondadori) Vivere in due. Diario di Clementina ${ }^{7}$. La protagonista es una mujer volcada en los cuidados del marido ingeniero y los niños. Banti (1953) lo cita como ejemplo de una publicación perniciosa por su adherencia a los valores y los esquemas sociales del Ventennio:

Chi vada a rileggersi, per esempio, quel Diario di Clementina che Vanda Bontà andava settimanalmente pubblicando su una rivista di mode fra il ' 40 e il ' 43 , non potrà fare a meno di sentirsi stringer alla gola da un nodo di disgusto. Era un confidarsi dismesso, non privo di abilità donnesca, della moglie del «soldatino» che coi teneri figli ne aspetta il ritorno vittorioso che la dispenserà del lavoro fuori casa, questa croce dei benpensanti del regime. Libri cosiffatti hanno più nociuto al nostro costume che una pubblicazione sfacciatamente immorale. (Banti 1953: 33)

6 Cino Del Duca, empresario, editor y productor cinematográfico, che ha empezado su carrera como comercial de novelas por entregas, ha sido un gran conocedor del mundo editorial destinado a las mujeres. Junto con los hermanos Alceo y Domenico, Del Duca intuye el enorme potencial comercial de las nuevas publicaciones populares: el cómic, in primis, y luego las fotonovelas, normalmente de tipo sentimental, en las que se especializará. Está considerado como una de las figuras fundamentales para la difusión de la cultura de masa en Europa (Antonutti: 2015).

$7 \quad$ En 1943, la editorial Sonzogno publica la versión integral. 
A través de la historia de Clementina, Wanda Bontà reparte consejos sobre cómo llevar la vida familiar y la narración parece ser efectivamente solo un pretexto para instruir a las lectoras en la paciencia y la dedicación para llegar a ser un perfecto ángel del hogar, sensato y sonriente (Teardo 2009; Vinall; Baranski 1991).

Sin embargo, se percibe siempre al fondo del relato un sentimiento de solidaridad femenina: tanto la escritora como sus lectoras parecen conscientes de las dificultades que conlleva el papel que la sociedad les ha impuesto, es decir garantizar el equilibrio y el bienestar de la familia sin sobresalir de una posición de segundo plano, a veces a lado de hombres autoritarios y prepotentes. La solución propuesta es siempre la misma: ser paciente y, cuando sea necesario, concederse alguna pequeña e inocente diversión (Rocella 1998: 84). Para una panorámica sobre la obra de Bontà y sus personajes femeninos véase Suadoni (2018).

La última novela de Bontà, Amanti (Valsecchi), una historia mucho más madura y melancólica que las anteriores, aparece en 1953. En total publica veinticinco títulos, tocando también la literatura infantil: Una corsa in paradiso y Paglietta (publicado por Vallardi, 1934) e I cagnolini di Perlarosa: racconto allegro per i piccoli (publicado por Genio, 1944).

\section{El rosa pedagogico para adolescentes: Signorinette y Signorinette nella vita.}

La narrativa de Wanda Bontà está centrada en un riguroso realismo. Incluso en la escritura: su prosa es lineal y casi humilde y muy alejada de "l'ostinato antirealismo" (Ricci 2017: 92) de Liala ${ }^{8}$. Los relatos de Bontà, cuya biografía es bastante más modesta que la aventurera y glamurosa de Liala ${ }^{9}$, se ciñen a una realidad poco llamativa con la que sus lectoras puedan identificarse más que soñar y su estilo parece celebrar un ideal de belleza sencilla que sube de tono solo en las descripciones de valores familiares o patrióticos.

También el relato de las historias de las adolescentes protagonistas de Signorinette y Signorinette nella vita se fundamenta en un realismo pedagógico que pretende instruir a las lectoras, jóvenes en este caso, además de entretenerlas y emocionarlas.

Signorinette es el libro más conocido de Wanda Bontà y, como hemos comentado, es un producto editorial construido rápidamente para un público que la escritora conocía muy bien. En la presentación de la edición de $1969^{10}$, Bontà confiesa su satisfacción por poder abandonar los esquemas narrativos románticos de las novelas sentimentales y dedicarse a una escritura más libre, casi una trascripción impulsiva de sus recuerdos de adolescente, con una historia enmarcada en un ambiente burgués, sólido y poco glamuroso que la autora describe con su habitual realismo.

\footnotetext{
8 Para una una panorámica sobre la legua de la novela rosa véanse Fresu (2016) y Ricci (2017).

9 En Liala los personajes, las historias, la ambientación y la prosa están inspirados en un ideal estético dannunziano: la escritora afirma explícitamente que no quiere relatar la cotidianidad, la pobreza, la mezquindad del día a día (Pozzato 1979: 97-98). Sus historias se desarrollan en ambientes aristocráticos o de la alta burguesía. El estilo "rifugge il corrivo e l'ordinario e ambisce, viceversa, a un tono evasivo e nobilitante, non di rado con esiti di libresca iperletterarietà" (Ricci 2017: 91).

10 Signorinette y Signorinette nella vita fueron publicados por primera vez por la editorial Mursia de Milán en 1938 y en 1942, respectivamente. La misma editorial reeditó ambas obras en 1969.
} 
El resultado es una obra dotada de cierta ligereza en la que el intento pedagógico no consigue ensombrecer completamente la atmósfera de novela de formación.

Posiblemente inspiradas en las Mujercitas de Louisa May Alcott (Calamita 2013; De Leeuw 2017), las protagonistas, Renata, Iris y Paolona (que muere al final del primer libro), son tres adolescentes de quince años que van al instituto. La historia se desarrolla en Milán, "la Nuova York peninsulare" (Banti 1953: 32). Iris, menuda y tímida, tiene aspiraciones literarias: "- Quindici anni! - La mamma sospirò. Iris ne dimostrava dodici, così piccina e fragile, con i capelli lisci che le cadevano sempre dinanzi al viso e gli occhi cerulei come 'ti scordar di me'" (Bontà 1991: 12). Renata es guapa y exuberante: "la mamma [...] non poteva fare a meno di pensare che quella sua figliola era sbocciata alta e perfetta come un giglio e che la zazzera nerissima le diffondeva intorno al volto un alone adorabile" (Bontà 1991: 15). Paolona es obesa, torpe e insegura:

Il signor Calocero sorrise. Quella grossa bambolona con le trecce riscuoteva tutte le sue simpatie. Assomigliava un po' alle giovanette formose della sua giuventù; e, costretta dalla sua grossezza a vestire semplici bluse e sottane non troppo attillate, gli aveva sempre destato in cuore una tenerezza fatta dei ricordi più cari; la madre, le sorelle giovanette, la fidanzata che poi si era sposata un altro" (Bontà 1991: 48).

En la descripción física de las protagonistas emerge la inclinación hacia una nueva idea de feminidad (Sani 2014: 73): se celebra el aspecto de Renata, una belleza moderna que recuerda a la Signorina Grandi Firme di Gino Boccasile ${ }^{11}$, aunque sea un ideal estético rechazado por el fascismo, mientras que la figura rolliza de Paolona, lejos de evocar a la mujer lozana y rural muy querida por el ideario fascista, le impide llevar una vida normal y saludable.

Falta en las protagonistas de Signorinette la perfecta adherencia al ideal femenino del ventennio fascista, que caracteriza a Clementina: las vivencias y el carácter de Clementina tienen que ser ejemplares para que sirvan de modelo y puedan propiciar la ocasión para dar consejos a las lectoras. Los personajes femeninos de las dos entregas de Signorinette, en cambio, no son solo construcciones literarias finalizadas al aleccionamiento del público; el sentimiento de solidaridad femenina que, como dijimos, hace de trasfondo a los episodios que componen Vivere in due, cobra en Signorinette el mismo relieve de la finalidad pedagógica, revelando por parte de la autora un sentimiento de afectuosa comprensión hacia la inmadurez de las adolescentes, todavía incapaces de enfrentarse con la realidad.

Sin embargo, sentido común y realismo siguen siendo calidades imprescindibles para evitar perderse. En un episodio del primer libro, Renata acepta citarse con un actor de teatro, Marcello Lancia, porque sueña un futuro distinto del de madre de familia:

11 Gino Boccasile, ilustrador y pintor italiano, creó una serie de ilustraciones a color de figuras femeninas para las cubiertas de la revista Grandi Firme, publicación periódica fundada y directa por Pitigrilli. 
- [...] E devi sapere che sogno il mio avvenire molto diverso da quello che vorrebbe papà.

- Cioè?

- Lusso strepitoso, viaggi per il mondo, ballare di notte e dormire di giorno. Non vorrei sposarmi, insomma, o tutt'al piú ricorrere al matrimonio quando avrò venticinque anni. Capisci?

- Non afferro bene.

- Insomma -sillabò Renata- ci-ne-ma-to-gra-fo! (Bontà 1991: 39)

Y frente a la reacción perpleja de Iris declara: "Oh, Iris, del resto tu mi conosci. Allegra, spensierata, ma il giudizio non mi manca. Furbe come me ce ne sono poche, e saprò come comportarmi, vedrai" (Bontà 1991: 40).

Renata descubrirá más adelante las verdaderas y poco nobles intenciones del actor y su exuberancia se verá castigada con una pequeña humillación:

-Vedi, babbo, sono già stata molto punita. Mi credevo bella e affascinante, e invece sono stata corteggiata soltanto per la mia probabile dote.

Di nuovo la fanciulla alzò gli occhi su suo padre; ed erano occhi mutati, occhi che le lacrime avevano aperto su ciò che è sogno e su ciò che è realtà (Bontà 1991: 222).

La dicotomía sueño-realidad se hace explícita y el mensaje parece claro: más que los grandes ideales y los sueños cuenta la capacidad de "saber cómo comportarse".

De hecho, parece que la historia de Gisella, una nueva compañera de clase, haya sido incluida en la narración como una advertencia: "Abbiamo una nuova compagna di scuola. Che tipo! Capelli ossigenati, quasi rossi. Dicono che sia interessante, ma a me pare volgare. Unghie rosse, labbra rosse. L'hanno già chiamata in Direzione per questo". (Bontà 1991: 35). Se oyen rumores sobre ella: se dice que se ha escapado de casa, que vive sola y que en su casa hay un continuo vaivén de chicas, e incluso de chicos. También en este caso el juicio de la autora no es demasiado duro: Gisella no es un personaje completamente negativo pero no sabe comportarse como debido y el resultado es una reputación equívoca, un matrimonio pobre y un futuro incierto.

En el primer libro la narración se desarrolla con el relato de la cotidianidad de las chicas: la preparación del examen de matemáticas, la clase de educación física, las visitas al dentista... Faltan eventos extraordinarios, pasiones perturbadoras. Las primeras inquietudes sentimentales se tratan como ingenuidades y vienen descritas con una condescendencia casi materna. En la escena siguiente Paola y Renata se encuentran en la sala de espera del dentista:

- [...] Io...io sono una ragazza seria. E se a te piace far già la signorina e parlar forte quando passa il professore di musica, per farti guardare...

- Cos'è questa storia? Non si può farsi guardare? Abbiamo due anni forse?

- Sì, io sono una bambina e voglio restare una bambina. Gioco con la bambola e vado ancora in braccio alla mamma.

- Brava... la sfonderai! (Bontà 1991: 18) 
Solo a Iris (posiblemente alter ego de la escritora) se le concede el lujo de aspirar a una vida distinta de la exclusivamente dedicada al matrimonio, la maternidad o, como máximo, un trabajo como profesora. Iris escribe poesías y al final de Signorinette nella vita publicará una novela. Sin embargo ella también tiene que enfrentarse con la realidad y acostumbrarse a la idea de que un matrimonio con un oficial de buena familia, aun sin una grande implicación romántica, es preferible a la pasión inquieta por un joven y sombrío submarinista que declara, desde el principio, que no desea tener vínculos sentimentales.

El enfrentamiento entre modernidad y tradición no llega a cuestionar valores fundamentales como la maternidad que tiene que ser dedicación exclusiva a los hijos:

Le mamme debbono amare, debbono dare, pensando sempre che le loro figliole le amano, che le loro figliole non potrebbero vivere senza di loro. Guai a dubitarne un momento solo. Il cuore pare fermarsi... perché le mamme, ormai non aspettano più nulla dalla vita, vivono soltanto perché ci sono delle piccole Iris nella loro casa... (Bontà 1991: 54).

Signorinette nella vita se publica en 1942. La coincidencia de su elaboración con el ingreso de Italia en la segunda guerra mundial hace que la prosa de la autora se adecue en algunos pasos al tono solemne de la propaganda. Las nuevas historias de Renata e Iris, a las que se añade Ermelinda, intentan reproducir la atmósfera de comedia adolescente presente en la primera entrega, como declara Bontà en la presentación a la edición de 1969:

Le prime pagine di Signorinette nella vita rispecchiano, con la loro forzata allegria, la lotta che dovevo sostenere contro la stanchezza e lo sconforto. Mi dicevo infatti che non dovevo rattristare le mie giovani lettrici; meglio narrar loro una storia piacevole e un po' distaccata dalla dura realtà. Ma com'era difficile! (Bontà 1969: 5)

Las señoritas han crecido y tienen que enfrentarse con problemas muy concretos que ya no dejan espacio para fantasías:

Riccardo era il fidanzato, un giovane molto simpatico che aveva ben avviato uno studio di dentista. Esigeva che Renata si preparasse al matrimonio con molta serietà. Renata invece detestava la serietà. Le piaceva andare in bicicletta a fianco di Riccardo, ballare con lui e immaginare la sua vita coniugale come un gioco molto divertente. Sarebbe andata ad abitare in un quartiere un po' lontano dalla mamma, che era un tesoro di donna, ma la subissava di consigli assennati. Da quando Renata si era fidanzata, non faceva che sottoporle ricette di cucina. Renata non la intendeva così; Riccardo sposandosi con lei avrebbe certo mangiato qualche cosa di buono, ma le cuoche sono fatte apposta per questo e poi ci sono le rosticcerie. (Bontà 1969: 9)

Como en el primer episodio de Signorinette, la resistencia de Renata a convertirse en una perfecta mujer y madre es tratada con cierta benevolencia por la autora, así 
como a veces se notan los rasgos caricaturales del novio celoso, protector y paternalista en el personaje de Riccardo, el novio de Renata, que teniendo que ir a la guerra, quiere organizar la vida de la chica: "Partirò fra venti giorni e desidererei vivamente che quest'anno Renata non passasse due mesi al mare. Sarò forse esigente, ma pensarla sempre sulla spiaggia, tra tanti farfalloni..." (Bontà 1969: 16). Y más adelante, cuando Renata declara que desea participar en la guerra como enfermera:

Nemmeno per idea, cara. Le ragazze come te stanno bene a casa loro. Anche se tu andassi in un nido di bambini, insegneresti loro soltanto a toccarsi il naso con la lingua! Se tu mi vuoi bene davvero, dovrai stare il più possibile vicino a tua madre e alla mia. Studierai, mi scriverai tutti i giorni e mi aspetterai come una brava donnina (Bontà 1969: 16).

La guerra está necesariamente presente en la narración, así como referencias, directas o indirectas, al valor del patriotismo. De hecho, a pesar de que la caída de la dictadura sea inminente, el clima bélico hace que el ideario fascista encuentre más espacio en este segundo libro que en el primero. Es el caso, por ejemplo, de la exaltación de la importancia de la actividad física (Canella; Giuntini 2009; Coronado 2017): en Signorinette nella vita dos capítulos están dedicados a un campamento deportivo al que asisten las protagonistas. Abundan en estas páginas las descripciones de los rituales cotidianos de ejercicio físico y la organización casi militar de la jornada que empieza con el izamiento de la bandera:

In gonna pantaloni blu e camicetta di un biancore smagliante, si avviarono verso lo spiazzo delle esercitazioni. In un silenzio così profondo che si sarebbe sentita volare una mosca, le ragazze si schierarono in due squadre [...]. Un piccolo scatto alla corda, e a poco a poco, come una fiamma che divampa mano a mano che morde il legno, il vessillo si elevò e si allargò palpitante nel sole, contro il cielo di cristallo (Bontà 1969: 178).

\section{Conclusiones}

A pesar de la extrema heterogeneidad (por calidad literaria, estilo o ideología) de las obras que pueden ser etiquetadas como rosa, hay un denominador común que se encuentra en su estatus de producto de masa, receptivo de las inquietudes de su público y en el que el deseo y las aspiraciones femeninas encuentran su espacio sin verse humillados ni necesariamente sublimados en la maternidad (Sani 2014).

La propaganda fascista construye dos modelos femeninos antitéticos en los que se unen valores sociales y estéticos: exalta la donna-madre, patriótica, fuerte, tranquila y prolífica contra la donna-crisi, cosmopolita, urbana, magra, histérica, decadente y estéril, resultado de la nefasta influencia de la modernización (Coronado 2009). Y si el rosa moderadamente transgresivo y anti-realístico de Liala ofrece a las lectoras una evasión a través de ambientes, situaciones y personajes socialmente inalcanzables, el 
realismo pedagógico de Wanda Bontà, que en Vivere in due había construido una narración edificante en la exaltación de los valores tradicionales, propone en Signorinette el enfrentamiento entre modelos femeninos tradicionales y ambiciones de emancipación. Estas últimas se corresponden principalmente con el rechazo a la figura de ama de casa relegada exclusivamente al cuidado de la familia y vienen tratadas con comprensión y condescendencia por parte de la autora que no las condena aunque obliga a sus personajes a quedarse bien ancladas a la realidad. A pesar de ello, Bontà no prescinde en Signorinette de algunos de los pilares fundamentales de los ideales fascistas (el patriotismo, la celebración de la actividad física, los valores familiares).

Las intenciones educativas en los dos libros de Signorinette se resuelven en el intento de la escritora de guiar el acercamiento a la madurez de las jóvenes lectoras recordando la importancia de atenerse a algunas normas de comportamiento para llegar a obtener un estatus económicamente y socialmente decente y haciendo referencia a una realidad concreta con la que sea fácil identificarse, lejos de los ambientes y las tramas extremas o glamurosas relatadas en las novelas rosa transgresivas. De hecho, la descripción del mundo en el que se mueven las protagonistas proporciona una información valiosa sobre la condición de la mujer y sobre sus intentos, a veces tímidos, otras veces imprudentes, de emanciparse de los modelos femeninos tradicionales.

El hecho de estar destinado a un público de adolescentes, le permite a Wanda Bontà de alejarse a menudo de las situaciones y los clichés típicos del rosa, dedicando largo espacio a las inquietudes personales de las protagonistas. La transición de la niñez a la edad adulta de sus señoritas está contada con afectuosa y solidaria participación y con explícitas referencias a los esquemas sociales, muchas veces humillantes, impuestos por un ideal femenino que se trata frecuentemente con ironía: "Non scocciare, Ermelinda. Io sono una donna e mi servo di piccoli mezzi; lui è un uomo e può concedersi il lusso di essere nobile e stoico" (Bontà 1969: 193).

\section{BIBLIOGRAFÍA}

ÅKERSTRÖM, Ulla (2018):"“'Sambadù, amore negro' di Mura. Censura fascista e sfida alla morale nell'Italia di Mussolini”. Romance Studies. 36 (3): 101-110.

ANTONUTTI, Isabelle (2015): Cino Del Duca. Un editore tra Italia e Francia. Milano: Editrice Franco Angeli.

BANTI, Anna (1953):"Storia e ragioni del 'romanzo rosa"”. Paragone. IV (38): 28-34.

BALESTRINI, Nanni; GIULIANI, Alfredo; BARILLI, Renato; GUGLIELMI, Angelo (2003): Gruppo 63. L'antologia. Critica e teoria. Milano: Bompiani.

BONTÀ, Wanda (1969): Signorinette nella vita. Milano: Mursia.

(1991): Signorinette. Milano: Mursia.

BRAVO, Anna (2011): Il fotoromanzo. Bologna: Il Mulino.

CALAMITA, Francesca (2016) "Bird-Like-Eating Attitudes, Fat-Shaming and Ideal Body Shape in Italian Women's Writing of the 1930s-1940s", (Tumini, Angela; Wagner, Tim; Alpanes, Amparo) Bridges Across Cultures Conference Proceedings. Perugia: Voces del Caribe, 60-67. 
CANELLA, Maria; GIUNTINI, Sergio (2009): Sport e fascismo. Milano: Franco Angeli.

CORONADO RUIZ, Carlota (2009): Esposa y madre ejemplar: la maternidad en los noticiarios Luce durante el fascismo (1928-1943). Madrid: Editorial Complutense.

CORONADO RUIZ, Carlota (2017): "Deporte femenino en Italia entre el fascismo y la democracia (1927-53)". Revista Estudos Feministas, Florianópolis, 26 (1): 1-17.

CROCE, Benedetto (1940): La letteratura della nuova Italia. Saggi critici. Vol: IV. Bari: Laterza.

DE FEO, Luca (2012): Case editrici: Femminile plurale (Tesis doctoral). Universidad de Sevilla, Sevilla.

DE LEEUW, Cleo (2017): Worlds Apart? Wanda Bontà's Signorinette (1938), an Adaptation of L.M. Alcott's Little Women (1868)? (Trabajo fin de máster). Universiteit Gent, Gent (Bélgica).

DETTI, Ermanno (1989): Le carte rosa. Firenze: La Nuova Italia.

FAETI, Antonio (1980): Dacci questo veleno: fiabe, fumetti, feulletons, bambine. Milano: Mondadori.

FRESU, Rita (2016): L'infinito pulviscolo. Tipologia linguistica della (para) letteratura femminile in Italia tra Otto e Novecento. Milano: Franco Angeli.

GRAMSCI, Antonio (1975): Quaderni dal carcere. Vol: I. Torino: Einaudi.

MIRABILE, Andrea (2005): Parola e immagine nel Novecento italiano: l'ekphrasis in Longhi, Banti, Pasolini e Testori (Tesis doctoral). Universily of North Carolina, Chapel Hill, North Carolina (U.S.A.).

MORREALE, Emiliano (2011): Così piangevano. Roma: Donzelli Editore.

POZZATO, Maria Pia (1979): "Liala". Carolina Invernizio, Matilde Serao, Liala. Firenze: La Nuova Italia: 95-122.

POZZATO, Maria Pia (1982): Il romanzo rosa. Milano: Espresso Strumenti.

RICCI, Laura (2017): Paraletteratura. Lingua e stile dei generi di consumo. Roma: Carocci.

ROCELLA, Eugenia (1998): La letteratura rosa. Roma: Editori Riuniti.

SANI, Filippo (2014): “Wanda Bontà: 'L'Intrepido' et 'Signorinette'. Aux origines de la 'littérature rose' pédagogique". Annali di storia dell'educazione e delle istituzioni scolastiche. 21: 69-77.

SUADONI, Anna (2018): "Wanda Bontà e gli stereotipi femminili nella letteratura rosa della prima metà del Novecento". Querelle des femmes. Male and female voices in italy and europe. Szczecin: Volumina: 449 - 456.

TEARDO, Sara (2009): Alla conquista della scena: donne e scrittura negli anni Cinquanta e Sessanta (Tesi dottorale). The State University of New Jersey, New Brunswick, New Jersey (U.S.A.). Recuperado en https://rucore.libraries.rutgers.edu/rutgers-lib/25946/PDF/1/playl, 20 luglio 2018.

VINALL, Shirley W; BARANSKI, Zygmunt G (1991):Women And Italy: Essays On Gender Culture And History. Lussemburgo: Springer. 


\section{PERFIL ACADÉMICO Y PROFESIONAL}

Licenciada por la Università La Sapienza de Roma, especialización en lingüística. Doctorada en 2014 por el Departamento de Lingüística General y Teoría de la Literatura de la Universidad de Granada. Profesora, en la actualidad, en el Departamento de Filología Románica de la Universidad de Granada, en el área de italiano. Mis intereses investigadores giran en torno a la lengua italiana, la lingüística descriptiva, la lingüística aplicada a la enseñanza de idiomas y la paraliteratura. He realizado publicaciones sobre lingüística italiana y contrastiva español-italiano. Desde 2016 me ocupo también de edición de textos. Además he cursado un máster en edición de UCM-Santillana y he colaborado con editoriales realizando traducciones e informes de lectura.

Fecha de recepción : 01/02/2019

Fecha de aceptación : 21/03/2019 